DIGITALCOMMONS

@WAYNESTATE -
Michigan Journal of Counseling:

Research, Theory and Practice

Volume $41 \mid$ Issue 1

Article 4

$3-1-2014$

\title{
Outlining a Crisis Management Plan for a Community: Crisis Planning in Michigan
}

Nicholaus Erber

Walden University

Follow this and additional works at: https://digitalcommons.wayne.edu/mijoc

\section{Recommended Citation}

Erber, N. (2014). Outlining a Crisis Management Plan for a Community: Crisis Planning in Michigan, Michigan Journal of Counseling, 41(1), 38-49. doi:10.22237/mijoc/1393632180

This Article is brought to you for free and open access by the Open Access Journals at DigitalCommons@WayneState. It has been accepted for inclusion in Michigan Journal of Counseling: Research, Theory and Practice by an authorized editor of DigitalCommons@WayneState. 


\title{
Outlining a Crisis Management Plan for a Community: Crisis Planning in
}

\author{
Michigan
}

Nicholaus Erber

Walden University

\begin{abstract}
During a crisis, the environment is often chaotic and unpredictable, added to the already devastated people and systems suffering the crisis. Order and structure can provide a sense of safety and moving forward after a crisis for everyone involved in the recovery effort. Counselors utilize crisis intervention models combined with evidence-based counseling practices to implement recovery strategies for individuals, families, and communities on several scales.
\end{abstract}

\section{Outlining a Crisis Management Plan for a Community: Crisis Planning in Michigan}

Crisis management requires a crisis management plan, which is developed by a crisis management team, and which trained professionals implement in their respective areas of crisis response (Crandall, Parnell, \& Spillman, 2010; James \& Gilliland, 2013). During a crisis, the environment is often chaotic and unpredictable, added to the already devastated people and systems suffering the crisis. Order and structure can provide a sense of safety after a crisis for everyone involved in the recovery effort. Crisis response also requires crisis management teams to consider ethical, legal and multicultural challenges that are revealed during a crisis, which are often amplified versions of precrisis issues (Andrulis, Siddiqui, \& Gantner, 2007; Collins \& Pieterse, 2007; Consoli, Kim, \& Meyer, 2008). Counselors utilize crisis intervention models combined with evidence-based practices in order to implement recovery strategies for individuals, families, and communities on several scales. In this paper, I will discuss several crisis management issues related to planning and implementation that can be applied to small- and large-scale crises.

\section{Crisis Management Team Strategy}

Before a crisis management plan can be developed, a Crisis Management Team must be assembled from community leaders and stakeholders from all aspects of the community (Beaulieu, n.d.; Hildreth, 2007; James \& Gilliland, 2013; Massey \& Larsen, 2006). Leaders from various levels of society

Nick Erber is a doctoral student at Walden University in Counselor Education and Supervision. Nick works full-time as a counselor and coordinator of the Thomas Judd Care Center at Munson Medical Center in Traverse City, MI. Nick is currently working on his dissertation and plans to be done this winter. 
ideally compose a crisis management team, from local authorities to state and national agencies, and possibly international organizations. Local, state, national, and international levels of a crisis management plan can be developed to address crises and disasters of varying scope and size (James \& Gilliland, 2013). A strong crisis management team would have members from several stakeholder groups across organizations to address threats at appropriate levels.

A crisis management team at the community level would be developed with local leaders from businesses, healthcare, police, fire, social welfare agencies, local media, utility and telecommunications organizations (Beaulieu, n.d.). Business leaders can provide financial support and expertise in managing the cash flow of recovery funds from state, national, and international organizations and government (Crandall et al., 2010). Healthcare providers can be organized to provide medical and mental health interventions across the spectrum of healthcare. Police and fire can coordinate rescue and emergency management at the site of the disaster. Social welfare agencies can provide food, clothing, and shelter services through a coordinated effort. Lastly, local media, utility and telecommunications organizations can provide ways to maintain communication channels throughout the disaster and get the infrastructure back up. Community members should also be a part of the stakeholder group assembled to develop the crisis management team. Once the stakeholder group assembles, members of the crisis management team can be identified, and authority can be outlined based on various disaster scenarios.

A crisis management team at the state level would be developed in a similar fashion but would have a much broader scope of responsibility. A statelevel crisis management team would be made up of leaders from the same groups identified above, but the leaders would represent geographic regions, thereby adding to the diversity of the team (Hildreth, 2007). Organizations across a state will likely have access to a variety of important assets that could be useful in managing a statewide crisis. It is also likely that the state government body would manage a statewide crisis management team.

National crisis management teams exist already in the form of the Federal Emergency Management Authority and the various groups that fall under their purview in the United States. These also work in conjunction with international organizations such as the Red Cross and Amnesty International. These organizations are responsible on a national and global scale for catastrophic events that span states and countries and have the capability of organizing resources across large areas (James \& Gilliland, 2013).

\section{Organizational Resources}

Organizational resources are essential to the success of a crisis management plan. These resources vary based on location and scope of responsibility, and can be private, nonprofit, and government entities (Beaulieu, n.d.; Crandall et al., 2010; James \& Gilliland, 2013; Massey \& Larsen, 2006). Below are descriptions of each of these areas in detail. The general flow of resources is a top-down structure in that international organizations coordinate efforts with national organizations, and national organizations coordinate with state organizations, and finally state organizations coordinate with local entities. 


\section{Community}

Community resources are extremely varied and are controlled on a local level by community members. Examples of community resources are local food banks, churches, fire, police, utility companies, and healthcare facilities. These community resources can provide direct and immediate assistance if a local disaster were to occur. In a local disaster scenario, the local crisis management team would be activated to implement a crisis management plan that has a crisis management plan in place. If the crisis grows beyond the confines of the community and begins to affect a larger area, the community may receive state assistance and intervention to coordinate the crisis management plan.

In my local area, the crisis management team likely includes members from the city and county police, metropolitan and rural fire departments, local utility companies, food pantries, and the local hospital system. I currently work for a hospital and part of the orientation to working at the facility is learning about the levels of the crisis management plan in which the hospital may become involved. Local law enforcement and rescue agencies coordinate with the hospital at all levels of a disaster to provide comprehensive medical care during a disaster. The Traverse City community serves as a host to the United States Coast Guard base that would also provide local support during a crisis. Traverse City is on the Lake Michigan coast, which is part of the largest inland body of fresh water in the UNITED STATES, and maritime crises may occur that would involve the Coast Guard.

\section{State}

Michigan has several state agencies charged with the coordination of statewide disasters; among them are the Michigan State Police, Michigan National Guard, United States Coast Guard, Michigan Disaster Response and the State Emergency Response Commission. Because of the Great Lakes, Michigan is unique in that we have a strong United States Coast Guard presence that integrated into the state resources. Michigan also has local chapters of the United Way, Salvation Army, Goodwill Industries, and Red Cross that would provide resources for a statewide crisis.

\section{Human Resources}

Disaster preparedness is a process by which local, state, and national organizations develop disaster response plans prior to a major disaster occurring (Scoppetta, 2008). In Michigan, the Michigan Crisis Response Association (MCRA, 2012), Michigan Partners in Crisis (MPC, 2012), and Third Level Crisis Center (n.d.) work together on local and state levels to address a wide range of crisis, including environmental, mental health, and other disasters.

The MCRA is a cooperative of 56 registered teams of individuals from law enforcement, fire and emergency medical services, hospital staff, clergy, educators, and mental health professional spread across the state. The organization is a private nonprofit that uses critical incident stress management (CISM) strategies to prepare for and respond to statewide crises. The MCP, a coalition of several large mental health advocacy and service groups, serves as a mediator between the criminal justice system and people with mental illnesses. Judges, prosecutors, mental health professionals, and law enforcement officers are all part of the coalition to enhance the quality and clinically appropriate treatment and support services for adults and children across Michigan during a crisis. Lastly, Third Level Crisis Center is a private nonprofit organization in the town that I live and work that provides crisis intervention and response to 
any crisis or disaster that might occur in the northern 25 counties of the Lower Peninsula of Michigan. Third Level is mostly a crisis hotline for people experiencing mental health emergencies, including suicidal ideation and is available $24 / 7$ every day of the year.

These agencies were readily available via a simple Google search for crisis organizations in Michigan, so it is likely that each organization is aware of the other's existence; however, there is no identifiable way to know this by looking at the websites. The three organizations seem to overlap in terms of the disaster services each is capable of providing. What is apparent is that the MCRA is similar to a state-level version of FEMA and would respond to similar crises. The MPC and Third Level both seem to be focused on mental health emergencies and are likely available to provide this service during a disaster, but the primary focus is on day-to-day crisis services to people experiencing psychiatric emergencies.

\section{Ethical and Legal Issues}

\section{Ethical Challenges}

Counseling ethics are professional standards that go beyond obeying the law and provide guidelines for counselors to follow in professional practice. The American Counseling Association (ACA) code of ethics is the seminal document for professional counselors to follow when questions about ethical decisions come up in practice (American Counseling Association, 2005). Ethical decisions are not always easily made and may require consultation with other professionals and organizations, particularly during a crisis in order to insure an ethical response. Consultation and pre-planning can oftentimes mitigate ethical violations by individuals or organizations following a crisis (Crandall et al., 2010).

One ethical issue that may come up is counselor competency (Consoli et al., 2008; Crandall et al., 2010; Hermann \& Herlihy, 2006). The ACA code of ethics states that counselors must practice within their scope of education and not perform counseling duties outside of the areas the counselor has received training (American Counseling Association, 2005, C.2.a). The code of ethics also describes the procedures for termination and referral if a client requires treatment outside the scope of the original counselor's practice (American Counseling Association, 2005, A.11.b). During a crisis, a counselor may be required to provide counseling services that they would not normally provide, such as finding resources for shelter and food. Counselors may lose contact with, or have to terminate with, a client because of issues outside the counselor's control. The counselor must then make quick decisions if they are competent to provide the service and that they are able to make the commitment to the client that they will fulfill their professional duties.

Beneficence and sensitivity to diversity are two additional ethical issues that may arise for professional counselors during a crisis (Hermann \& Herlihy, 2006). Beneficence is the counselor's ethical mandate to keep the clients' needs above their own personal needs and provide the safe and therapeutic environment for counseling to be productive (American Counseling Association, 2005, A.1.a). During a crisis, a counselor may also be affected by the disaster, leaving the counselor to cope with his or her own response to the crisis while providing counseling to victims of a disaster. A commitment to diversity is also 
an ethical obligation to counselors and this may be an issue for a counselor from outside the affected area who travels to offer services. The counselor from another state or country may travel to provide counseling services, and they may not be familiar with the cultural issues of the affected area. This discussion is certainly not an exhaustive list of ethical issues counselors face during a disaster, but these are some important considerations counselors need keep in mind if they are called to a disaster.

\section{Legal Challenges}

Counselors who respond to disasters are not immune from legal issues arising from professional practice. Some legal issues should be addressed prior to a counselor responding to a disaster to mitigate the damages the counselor may incur. One such issue would be agency liability for the counselors who will be providing disaster response services in crisis areas (Hermann \& Herlihy, 2006). Counselors that respond to a disaster may be put in dangerous situations, and the counselor may be required to provide counseling services to people that are from populations that disagree with a counselor's personal beliefs, or the counselor may be required to provide services outside the scope of their normal clinical practice. Counselors must avoid causing harm and cannot discriminate against many social groups, but they also may be required to do these things either based on professional obligation or by an employer.

Counselors must also provide for safety for their clients and may be involved in economic recovery efforts after a crisis to manage the rebuilding efforts. Counselors must follow the ethical standards of their profession, but may also be required to terminate services because of economic factors of the recovery (Crandall et al., 2010). Counselors are legally obligated to remain in a therapeutic relationship with a client until the counselor and/or client decide to terminate the relationship because the work is complete or for other reasons, but economic factors are generally not accepted reasons to terminate abruptly with clients (Hermann \& Herlihy, 2006). Counselors must make conscientious decisions about their work with clients during a crisis to mitigate legal issues, and to maintain a therapeutic relationship with their clients.

\section{Diversity and Multicultural Issues}

Counselors work with multicultural challenges during the day-to-day work of being a counselor, and disaster response also requires counselors to be multiculturally competent to the populations they serve (Andrulis et al., 2007; Collins \& Pieterse, 2007; Consoli et al., 2008; Hermann \& Herlihy, 2006). Some pertinent multicultural challenges that counselors may encounter during disaster response efforts are a the impact of a crisis on a poor community or the cultural conflicts that may arise when diverse cultures collide after a disaster (Andrulis et al., 2007; Consoli et al., 2008). Counselors must make sure they are aware of the cultural groups they will be working with during the disaster response, and minimize cultural issues in providing counseling services.

A cultural issue that may come up for counselors during the aftermath of a disaster is the potential for cultural misunderstandings of the value of cultural artifacts and meaning of family (Consoli et al., 2008). Counselors may make several assumptions about how a person is coping with a disaster in terms of individual impact while the client may instead be concerned with the impact on the family or community. Another aspect may be that the counselor may en- 
courage clients to 'find their own path' but the culture may be more responsive to an authority making choices and providing assistance (Consoli et al., 2008).

Communication is a significant aspect of providing counseling and may be an issue during a crisis for counselors who are unfamiliar with the culture group affected by the crisis. Cultures develop their own vernacular and language, and the counselor may not be educated in the local practices and customs, which could lead to cultural misunderstandings and problems with providing professional counseling services (Whealin, n.d.). Communications technology and infrastructure may be also be affected by the disaster or may not have existed at all prior to the crisis event. Although the Stafford Act (2007) may provide some financial assistance to communities for disaster preparedness and response, some communities may remain isolated and cut off technologically for communication of a disaster, and this may create chaos despite the best efforts of the agencies providing assistance.

Bereavement is another issue that is culture-bound and varies based on several factors. Cultural dynamics explain the attitudes of people toward death, such as death-accepting, death-defying, and death-denying (James \& Gilliland, 2013). James and Gilliland (2013) describe cultures' experience of death in these terms to describe the variety of cultural reactions that individuals have toward death. A death-accepting culture would accept death as a transition in life and allow for public displays of the grief response. Cultures that are death-denying display characteristics such as not speaking about death, not speaking about people who have died, and making death rituals a private affair, including the grieving process. Death-defying cultures, such as the United States, rely on medical interventions to prolong life sometimes well past medical necessity to avoid feeling grief. Sociocultural structures exist in communities which are shaped by laws and regulations, institutional restraints, and the development of specialists in death and dying. Spirituality and religion provide meaning to life and some people experiencing a faith-finding epiphany after a crisis. Sometimes the disaster area is so large that it is difficult to narrow down the dynamics of bereavement, but some basic principles of grieving exist, such as the Kubler-Ross model of grieving that includes five stages - denial, anger, bargaining, depression, and acceptance.

The psychological response to the crisis will also vary by culture and could present more barriers to rebuilding (James \& Gilliland, 2013). There will likely be a significant grief response following such a large-scale disaster, and cultures vary in terms of how long grieving should last and how to cope with the grief of losing everything. Individualist cultures are self-reliant while collectivist cultures will rely on the community for help (James \& Gilliland, 2013). In an ideal world, the crisis response organization should allow communities to work through their own grief and move forward at their own pace, which are not always practical solutions. Navigating this psychological trauma landscape will require the skills of mental health professionals to assist in helping individuals and communities process the grief and rebuild their lives.

\section{The Hybrid Model of Crisis Intervention}

For training purposes, the hybrid model of crisis intervention is a solid model to conceptualize the chaos of a crisis, and provide a new counselor with the tools to intervene in crises at several levels. Two strengths of the hybrid 
model are first that it is not a linear model and second that the ecological/ contextual paradigm provides a foundation for the model, which looks at a crisis from a global perspective and in context (James \& Gilliland, 2013). Linear models are not sufficient to capture the depth and breadth of a crisis reaction, nor do they possess the flexibility necessary in a crisis environment that is constantly shifting and changing. This requires crises to be assessed in a global manner that takes several factors into focus, such as people, equipment, infrastructure, and communications.

There are seven tasks performed in the hybrid model, and they are not static and are not intended to be performed sequentially (James \& Gilliland, 2013). The steps include engaging/initiating contact, exploring/assessing the problem, providing support in several ways, examining alternatives, making plans, obtaining commitment and follow up. The steps are part of an iterative process while managing the crisis. This constant reassessment is the hallmark of a learning organization that possesses the capability to adapt to changing systems and environments (Crandall et al., 2010), which is exactly what defines a crisis. Counselors provide services within the framework of the hybrid model utilizing evidence-based practices for trauma and crisis work.

The first stage includes engagement with the client and the establishing the role of the counselor. This stage may be repeated with each client if there are multiple crisis-survivors. The second stage assesses the impact of the crisis along affective, behavioral, and cognitive parameters, as well as exploring intrapersonal, interpersonal, and systemic impact and needs. In the third stage, the counselor begins providing support following Maslow's hierarchy of needs by providing physiological support, followed by safety and lastly a sense of belonging (Best et al., 2008; James \& Gilliland, 2013). During the fourth stage, the counselor should attempt to find multiple alternatives to meeting the needs of the people in terms of meeting short-term goals, such as financial assistance or de-escalating a tenuous situation. The fifth stage of the hybrid model of crisis intervention begins when a plan is fully devised, and goals are set. The final two steps may be the two most important as these involve the counselor remaining to monitor the progress and provide additional interventions and follow-up work.

\section{Evidence-Based Practices}

During a crisis, the traumatic event can trigger posttraumatic stress disorder (PTSD) in the affected people (James \& Gilliland, 2013). PTSD-related treatment interventions are sometimes population specific and clinicians should utilize the appropriate intervention for each affected group is essential to proper mental health treatment. Children appear to respond best to interventions targeted on empowerment through the use of cognitive-behavioral techniques, reprocessing the event, and distinguishing between the crisis and "normal life" (Putman, 2009). All populations are more susceptible to suicide and should be evaluated for this regularly (James \& Gilliland, 2013; Schwartz \& Cohen, 2001). Safety contracting with survivors is an intervention to use with people who are experiencing suicidal thoughts, and have shown some potential to preserve life (James \& Gilliland, 2013). People with substance use disorders are a vulnerable population during a crisis, primarily because the substance use increases impulsivity, which can increase the likelihood of accidental overdose or suicide (Ford, Russo, \& Mallon, 2007; James \& Gilliland, 2013). The Trauma 
Adaptive Recovery Group Education and Therapy (TARGET) interventions have shown to positive outcomes of trauma survivors with substance use disorders (Ford et al., 2007). Responding to such a large-scale disaster means developing several treatment protocols for people from different backgrounds and experiencing various pre-disaster psychological conditions. Two additional evidence-based cognitive-behavioral treatment methods that can be used in crisis situations are motivational interviewing and prolonged exposure.

\section{Motivational Interviewing}

Motivational interviewing is a directive, person-centered therapy designed to move a client away from resistance to change and into a mindset where they are able to process change effectively (Miller \& Rollnick, 1991). Motivational interviewing is grounded in the transtheoretical model of change (Prochaska \& DiClemente, 1982), and the overall goal of therapy is to prepare a person to move into an active role in creating change in their lives. The stage of change the person is in at the time they are being treated informs the interventions that counselors use. Motivational interviewing requires the use of microskills during the session to empower the client to take more control, thereby diminishing anxiety and stress responses to trauma (Gerber \& Basham, 1999; Laureate Education, Inc., 2010).

Motivational interviewing requires the use of certain therapeutic skills during the preparation stage of treatment (Miller \& Rollnick, 1991), which is similar to the integration phase of crisis response (Laureate Education, Inc., 2010). In the preparation stage, the person is 'gearing up' for change by creating a plan that they intend to follow to alleviate the symptoms. This plan often includes behavioral changes that allow for more psychologically healthy behaviors. In the integration phase of crisis response, the person is beginning to process the crisis into his/her own experience of the world and prepares to begin taking action to resolve the crisis. People may not feel they can move forward, and the empowerment of $\mathrm{MI}$ techniques can help a person feel more prepared to process the crisis. Specific tasks the therapist uses in MI therapy are expressing empathy for the person through genuineness and positive regard; developing discrepancy between the person's present behavior and the broader scope of the crisis; avoid arguing with the person by using soft confrontation; rolling with resistance by reframing resistant statements that increase an understanding of flexibility, and cultivate self-efficacy in a person by maximizing confidence in the person (Gerber \& Basham, 1999). Motivational interviewing can be used to build rapport with a person who survived a crisis but who is in a psychological state not conducive to traditional therapy methods.

\section{Prolonged Exposure}

Prolonged exposure (PE) is an evidence-based therapy focused on processing traumatic events through memory recall and stress reducing interventions (National Center for PTSD, n.d.). There is strong empirical support for PE in working with people who have sexual assault, crisis events, and military trauma experiences in their lives that cause problems in day-to-day life. PE requires four specific interventions that a counselor uses when working with a client. The intent of $\mathrm{PE}$ is to normalize the traumatic experience in a person so that they can move forward with their lives and diminish intrusive thoughts, nightmares, and panic symptoms associated with crisis reactions.

The four PE interventions used with trauma survivors are psychoeducation, breathing skills, in vivo exposure, and imaginal exposure (National Center 
for PTSD, n.d.). The psychoeducation component of PE focuses on teaching people about how to process traumatic events and the reasons behind the exposure activities later in the therapy. In order to process the anxiety associated with the exposure, it is important to teach the person deep breathing skills to manage the anxiety. Once these two phases are complete, the exposure aspect of the therapy begins. This can proceed one of two ways, through in vivo (or real life) exposure in which a person participates in anxiety producing activities, in their day-to-day lives that they find anxiety producing, such as going to a place in their neighborhood to trigger an anxiety response. These are carefully designed in a hierarchical manner from less provoking to more provoking, and the person moves through the stages sequentially. Imaginal exposure is conducted in the counselor's office where the person goes through telling and retelling the traumatic experience while recalling vivid details during each retelling. The counselor coaches the person through each of these exercises to use the breathing techniques and focus on the sense of safety in the present moment.

\section{Vicarious Trauma and Burnout}

Counselors and other helping professionals are at a higher risk of experiencing trauma, burnout and compassion fatigue than the general population because of the nature of the work (James \& Gilliland, 2013; Musa \& Hamid, 2008; Trippany, White Kress, \& Wilcoxon, 2004). Over time, counselors will experience trauma from client stories of traumatic histories and experiences, known professionally as vicarious trauma (Fallahi \& Lesik, 2009; James \& Gilliland, 2013; Trippany et al., 2004). The impact of vicarious trauma on counselors can lead to what is commonly known as burnout, or compassion fatigue, and can diminish a counselor's capacity for empathy and effectiveness in counseling others (James \& Gilliland, 2013; Musa \& Hamid, 2008; Trippany et al., 2004). An example of this would be a counselor working in a crisis shelter who experiences client crises on a professional level as part of the daily work. Over time, the counselor may begin to have intrusive thoughts about the work and experience a diminished desire to help others and find excuses to avoid going to work.

Vicarious trauma and burnout can be managed and treated through simple interventions that a counselor can perform on himself or herself. First, acknowledging that there is a problem is a significant first step for counselors working on their own trauma (James \& Gilliland, 2013). Counselors can begin a multimodal intervention of self-care once the problem is acknowledged to manage and treat the vicarious trauma and burnout by engaging in activities that are therapeutic such as journaling, exercise, and hobbies. The constructivist self-development theory (CSDT) is a model that easily conceptualizes vicarious trauma that counselors experience by recognizing the importance of cognitive schemas the counselors form from the traumatic stories clients tell and the interaction of the schemas in the counselors psychosocial environment (Trippany et al., 2004). Counselors may use the CSDT comprehensive model to manage their own vicarious trauma.

\section{Crisis Management Plan Evaluation}

Evaluation of a crisis management plan is inherently humanistic with the idea of maximizing the preservation of life during and after a crisis with a solid crisis management plan (James \& Gilliland, 2013). Disasters result in death and destruction on a large scale that is often difficult for people to process. Multiple systems may be impacted starting with individuals and families, 
through local communication channels, and if the crisis is severe enough, into national media, government, and professional crisis intervention organizations (James \& Gilliland, 2013). For example, the recent bombing incident in Boston followed by the televised manhunt of the suspects. The media coverage provided the public with a hawk-eye view of the incident itself, and each step as the crisis plan was implemented and ultimately successful. This encourages support of society and can add value to the evaluation of the plan by including this element.

Secondly, the principles of crisis management plan development are valuable in the reevaluation of a plan once implemented. In a sense, going back to the building blocks of the plan once it is written to insure that all the necessary elements are covered in the plan is essential to the value of the crisis management plan itself. Principles such as an interdisciplinary approach, multitheoretical model, individuals as part of an ecosystem, context, time, meaning, efficiency, cooperation, a range of interventions and quality services (James \& Gilliland, 2013) combined can ensure that all the needs will be covered and can improve the success factor of the crisis management plan.

\section{Social Change Impact}

The social change impact in my community may be in the categories of multicultural competence and evidence-based practice. In my community, there are several crisis plans already in place because of the diligence of the community leaders to have these mechanisms in place. Part of the orientation to the organization is to become familiar with the crisis plans, and how the hospital fits into the crisis plans that involve the community and state. The plans I have seen are detailed and meet all the criteria outlined in this paper.

Multicultural competence is one area that could be improved in crisis plans. The population inside the town I live in is minimally diverse, and I understand through my employment that we have a dearth of language capable translators in our area, but there are minority populations of non-English speaking persons that could have difficulty in a crisis situation. Increasing the capability of the translation services or translators would benefit the community.

Additionally, the crisis plans include the use of mental health professionals to provide crisis intervention services. As a local member of a professional community, there are several CISD trained crisis-specialists in the area. There seems to be a lack of specialists trained in any other evidence-based intervention model, specifically in the hybrid model presented in this paper. An effective crisis plan would include the hybrid model and would require more training and improve service delivery by taking a holistic approach to crisis assessment and intervention.

\section{References}

American Counseling Association. (2005). Code of ethics. Washington, DC: Author.

Andrulis, D. P., Siddiqui, N. J., \& Gantner, J. L. (2007). Preparing racially and ethnically diverse communities for public health emergencies. Health Affairs, 26(5), 1269-1279. 
Beaulieu, L. J. (n.d.). Mapping the assets of your community: A key component for building local capacity. Retrieved from http://srdc.msstate.edu/ publications/archive/227.pdf

Best, D., Day, E., McCarthy, T., Darlington, I., \& Pinchbeck, K. (2008). The hierarchy of needs and care planning in addiction services: What Maslow can tell us about addressing competing priorities? Addiction Research \& Theory, 16(4), 305-307.

Collins, N. M., \& Pieterse, A. L. (2007). Critical incident analysis based training: An approach for developing active racial/cultural awareness. Journal of Counseling \& Development, 85(1), 14-23.

Consoli, A. J., Kim, B. S. K., \& Meyer, D. M. (2008). Counselors' values profile: Implications for counseling ethnic minority clients. Counseling \& Values, 52(3), 181-197.

Crandall, W. R., Parnell, J. A., \& Spillman, J. E. (2010). Crisis management in the new strategy landscape. Thousand Oaks, CA: SAGE Publications, Inc.

Fallahi, C. R., \& Lesik, S. A. (2009). The effects of vicarious exposure to the recent massacre at Virginia Tech. Psychological Trauma: Theory, Research, Practice, and Policy, 1(3), 220-230. doi:10.1037/a0015052

Ford, J. D., Russo, E. M., \& Mallon, S. D. (2007). Integrating treatment of posttraumatic stress disorder and substance use disorder. Journal of Counseling \& Development, 85(4), 475-490.

Gerber, S., \& Basham, A. (1999). Responsive therapy and motivational interviewing: Postmodernist paradigms. Journal of Counseling \& Development, 77(4), 418.

Hermann, M. A., \& Herlihy, B. R. (2006). Legal and ethical implications of refusing to counsel homosexual clients. Journal of Counseling \& Development, 84(4), 414-418.

Hildreth, R. (2007). Integrating emergency and disaster planning. National Civic Review, 96(4), 59-61.

James, R. K., \& Gilliland, B. E. (2013). Crisis intervention strategies (7th ed.). Belmont, CA: Brooks/Cengage.

Laureate Education, Inc., (Executive Producer). (2010). During a crisis - Counselor interventions and strategies [video media]. Baltimore, MD: Author.

Massey, 2Joseph Eric, \& Larsen, J. P. (2006). Crisis management in real time: How to successfully plan for and respond to a crisis. Journal of Promotion Management, 12(3/4), 63-97.

Miller, W. R., \& Rollnick, S. (1991). Motivational interviewing: Preparing people to change addictive behavior. New York: Guilford Press.

Musa, S. A., \& Hamid, A. A. R. M. (2008). Psychological problems among aid workers operating in Darfur. Social Behavior \& Personality: An International Journal, 36(3), 407-416. doi:10.2224/sbp.2008.36.3.407

National Center for PTSD, (Producer). (n.d.). Cognitive behavioral psychotherapies for PTSD [Flash presentation]. Washington, DC: Veterans Administration. Retrieved from http://www.ptsd.va.gov/professional/ ptsd101/course-modules/course-modules.asp

Prochaska, J. O., \& DiClemente, C. C. (1982). Transtheoretical therapy: Toward a more integrative model of change. Psychotherapy: Theory, Research, Practice, Training, 19(3), 276-288. doi:10.1037/h0088437 
Putman, S. E. (2009). The monsters in my head: Posttraumatic stress disorder and the child survivor of sexual abuse. Journal of Counseling \& Development, 87(1), 80-89.

Schwartz, R. C., \& Cohen, B. N. (2001). Risk factors for suicidality among clients with schizophrenia. Journal of Counseling \& Development, 79(3), 314.

Scoppetta, N. (2008). Disaster planning and preparedness: A human story. Social Research, 75(3), 807-814.

Trippany, R. L., White Kress, V. E., \& Wilcoxon, S. A. (2004). Preventing vicarious trauma: What counselors should know when working with trauma survivors. Journal of Counseling \& Development, 82(1), 31-37. 